\title{
CLIPPERS, chronic lymphocytic inflammation with pontine perivascular enhancement responsive to steroids: A challenge in neurological practice, clinical landmarks (Review)
}

\author{
ANY DOCU AXELERAD ${ }^{1,2}$, ALINA ZORINA STROE ${ }^{1,2}$, CRISTINA MIHAI $^{3,4}$, CORINA FRECUS $^{3,4}$, \\ DRAGOS CATALIN JIANU ${ }^{5}$, DANIEL DOCU AXELERAD ${ }^{6}$ and ANCA ELENA GOGU ${ }^{5}$ \\ ${ }^{1}$ Department of Neurology, Faculty of General Medicine, 'Ovidius' University of Constanta, 900470 Constanta; \\ ${ }^{2}$ Neurology Department, County Emergency Clinical Hospital 'Sf. Apostol Andrei', 900591 Constanta; \\ ${ }^{3}$ Department of Pediatrics, Faculty of General Medicine, 'Ovidius' University of Constanta, 900470 Constanta; \\ ${ }^{4}$ Pediatrics Department, County Emergency Clinical Hospital ‘Sf. Apostol Andrei’, 900591 Constanta; \\ ${ }^{5}$ Department of Neurology, 'Victor Babes' University of Medicine and Pharmacy of Timisoara, 300041 Timisoara; \\ ${ }^{6}$ Department of Kinetotherapy, 'Brainaxy Clinic', 900628 Constanta, Romania
}

Received June 14, 2021; Accepted July 14, 2021

DOI: $10.3892 /$ etm.2021.10625

\begin{abstract}
Chronic lymphocytic inflammation with pontine perivascular enhancement responsive to steroids (CLIPPERS) is a relatively newly discovered and characterized condition affecting the central nervous system (CNS) that involves the brainstem almost ubiquitously and that focuses primarily on the pons. Characteristically, CLIPPERS represents a combination of clinical symptoms related to the pathology of the brainstem in particular and has a characteristic appearance on magnetic resonance imaging (MRI), with punctate and curvilinear gadolinium enhancement 'peppering' the pons. The lesions can be viewed via neuroimaging with a predominance in the pons and adjacent rhombencephalic structures, such as the cerebellar peduncles, cerebellum, medulla, and middle brain. These lesions may also spread and appear in other areas of the brain such as the thalamus or white matter. As the name suggests, this clinical syndrome responds to immunosuppressive treatment based on glucocorticosteroids (GCSs), expressed as both clinical and radiological improvements. Support for this treatment is required for a long period, since exacerbations may occur without. Thus, the diagnosis of CLIPPERS has garnered increased attention from clinicians and the active exclusion of differential alternative diagnoses is needed. A specific biomarker of serum or cerebrospinal fluid (CSF) for this condition has not been discovered. The pathophysiology
\end{abstract}

Correspondence to: Dr Alina Zorina Stroe, Department of Neurology, Faculty of General Medicine, 'Ovidius' University of Constanta, 1 Aleea Universității Street, 900470 Constanta, Romania E-mail: zorina.stroe@yahoo.com

Key words: CLIPPERS, chronic lymphocytic inflammation, pontine perivascular enhancement, responsive to steroids, central nervous system of this condition is not fully elucidated, and it is not yet clear whether CLIPPERS is a disease in its own right or is the culmination of several already known syndromes. Clinicians and radiologists should pay close attention to this condition as it is relatively easy to treat and patients can benefit from the early introduction of GCSs, which can produce significant results through long-term immunosuppression. Taking into account previous reports in the literature, which currently include over 50 cases reported as CLIPPERS, this analysis addressed the clinical features, and paraclinical and differential diagnoses of cases described in the literature.

\section{Contents}

1. Introduction

2. Main characteristics of CLIPPERS

3. Cases from the literature

4. Additional information

5. Conclusions

\section{Introduction}

Chronic lymphocytic inflammation with pontocerebellar perivascular enhancement responsive to steroids is more commonly known by its acronym, 'CLIPPERS', and was introduced to the scientific world in 2011 (1). It is generally confounded with other diseases, and, since it has a peculiar treatment, it is particularly important to understand this specific disease.

The fundamental mechanisms underlying the pathogenesis of CLIPPERS have not been completely elucidated, and a neural autoantibody target has not been established. Treatment with corticosteroids is efficacious in treating the inflammatory segment of the disease, but persistent or permanent treatment of CLIPPERS using steroids is restrained by adverse or unwanted secondary effects $(1,2)$. 


\section{Main characteristics of CLIPPERS}

CLIPPERS syndrome is identified by a few clinical aspects and a particular typical neuroimaging feature: brain stem signs and symptoms (the most common are represented by ataxia, diplopia, facial paraesthesia), that are ameliorated with corticosteroids and have a poor prognosis when treatment is interrupted. MRI images of the pons show punctiform images with 'salt and pepper' aspects or curves with contrast absorption $(1,2)$. Biopsies of the pons have regularly revealed an infiltration of the perivascular spaces by $\mathrm{T}$ cells, that represents why this syndrome has been added to the class of the inflammatory diseases located in the brain stem $(3,4)$.

Patients with CLIPPERS syndrome have shown characteristic, but nonspecific, perivascular, mainly T-cell infiltrate that serves, at the first observation, also as evident pathological proof of other recognized infectious, inflammatory, or demyelinating diseases $(3,4)$.

However, the three mentioned aspects are adequately distinguishable in diagnoses without the use of biopsies to exclude alternative diseases located in the brain stem that demand differential diagnoses, including multiple sclerosis, neuromyelitis optica, neurosarcoidosis, Behcet's disease, Bickerstaff's encephalitis, primary lymphoma and primary vasculitis or infectious vasculitis of the central nervous system (CNSJ) and paraneoplastic syndrome. T-cell infiltration and the improvement with treatment using corticosteroids indicate that the presently unidentified origin is an immuno-mediated source (5).

In the literature, it has been theorized that, instead of representing a disease, CLIPPERS may represent a syndrome with various causes $(2,3)$. In scientific reports, in a few situations, Clippers' syndrome has progressed to B-cell lymphoma (4) and has been hypothesized to be an infrequent cause of lacunar infarcts (6).

Numerous cases in the literature, from which the constitutive elements of the syndrome's description were consolidated, have demonstrated and postulated that, during immunotherapy suppression, the disease worsens and that therapy consisting of a minimum dose of $20 \mathrm{mg}$ of prednisone daily is recommended in order to prevent this worsening (7).

CLIPPERS is possibly underdiagnosed, and patients may be diagnosed and treated for another disorder for some time before re-diagnosis. In order to avoid misdiagnosis, increased understanding of this clinical-radiological syndrome is particularly significant, not only due to the beneficial response to corticosteroids, but also for the reduction in the number of diagnostic investigations, such as brain biopsy, which present high risks but are recommended in select cases (5-7).

\section{Cases from the literature}

We performed a narrative review of the literature and reviewed studies about CLIPPERS syndrome to describe the symptomatology and the prevalent cases in the literature. MEDLINE and ScienceDirect were searched for original articles and review of literature published between January 2011 and February 2021 describing studies that involved patients and results observed on the CLIPPERS syndrome symptomatology, imagistic findings, and presentations of the cases. The key words used for the search were: 'CLIPPERS syndrome' and 'chronic lymphocytic inflammation with pontine perivascular enhancement responsive to steroids'. We have included 45 articles in our study. The inclusion criteria was the presence of CLIPPERS syndrome, and the exclusion criteria was the absence of CLIPPERS syndrome. Our study included 45 articles.

The main clinical features described in the literature (7-11) are subacute progressive ataxia and diplopia. The radiological features are described as follows: numerous bilateral punctate or nodular enhancing lesions in one of the three following anatomical locations, pons, middle cerebellar peduncle and cerebellum, along with other single radiological lesions that may be punctiform or small in size but may unite in order to constitute more preponderant lesions (7-12).

Additionally, the lesions can be located in the spinal cord, basal ganglia, or cerebral white matter but may be less consist with growing distance from the pons (11-13). Responsiveness to corticosteroid treatment is rapid and especially important when both clinical and radiological responses are present (11-15).

The histopathological features include white matter perivascular lymphohistiocytic infiltrate with or without parenchymal extension (11-15). The infiltrate consists predominantly of: $\mathrm{CD}^{+}$and $\mathrm{CD} 20^{+}$lymphocytes. Notably, the following histopathological features are absent: monoclonal or atypical lymphocyte populations and necrotizing granulomatomas or giant cells, and histological features of vasculitis, which are important in excluding differential diagnoses (10-15). The differential diagnosis is conducted against demyelinating diseases, CNS lymphoma, Behcet's and Sjogren disease, glioma, primary CNS vasculitis, paraneoplastic syndrome, sarcoidosis, lues in the nervous system, Whipple's disease, tuberculosis, and histiocytosis (10-15).

The mean age at onset is 50 years (range 13 to 86 years), and prevalent studies from the literature have revealed a male preponderance with a sex ratio of 3:1. Regarding the patients that did not follow chronic corticosteroid therapy or immunosuppressive agents, CLIPPERS was demonstrated to have a relapsing remitting course (15-17). In the period of attacks, even though clinical and neurological amelioration following high doses of corticosteroids was consistently recognized, patients may present with impairments consisting of cognitive deterioration among the hindbrain, the spinal cord and even the cortex. In those cases, because of the lack of disease evolution, the clinical and radiological sequelae were associated with former attacks that were severe or untreated. Accordingly, preserving the disease within remission might avoid intensification of impairment. The scientific and medical world has decided, following observations from the case reports, that relapse does not occur as often or at all, in cases in which chronic corticosteroid therapy was managed exceeding $20 \mathrm{mg}$ per day $(8,9)$.

In the cases presented in Table SI $(3,8-39)$ some patients experienced non-CLIPPERS symptoms. A severe headache was the first symptom described by the patient reported by Buttmann et al (14) and one patient of the series of studies by Simon et al (10) developed headaches as the first symptom as well.

Additionally, in the evolution of the disease, some patients experienced hemorrhages, such as for the patient studied by Buttmann et al (14) who presented subacute bleeding in the 
right cerebellar white matter surrounded by a large perifocal edema with a minimal increase in ventricular size. Additionally, in the case report presented by Blaabjerg et al (12), the patient responded to steroid treatment but then had a fatal brainstem hemorrhage.

Lesions may be situated in the spinal cord, basal ganglia or cerebral white matter. The perivascular gadolinium enhancement pattern and steroid responsiveness suggest an autoimmune/inflammatory nature of this condition (3,17-19).

A distinct captivating and unidentified characteristic includes perivascular inflammation that affects the pontine and peripontine areas, which implies that the disease has a specific territorial immunological aim. Definitive immunological and histological studies should be conducted to target this fact. Kastrup et al (20) revealed the presence of enhanced IgE levels in two of their patients, indicating that the inflammatory mechanism in CLIPPERS syndrome can be due to an allergic process. CLIPPERS syndrome represents a novel disease involving the CNS, for which the features have yet been clearly defined (20).

Kastrup et al (20) reported that, in patients, ataxia and perioral paresthesia appear to be characteristic of the symptomatology, and histological exams did not reveal evidence of demyelination. Neuropathological investigations showed a significant angiocentric but still dispersed infiltration sequence of the CNS parenchyma in small mature T-lymphocytes. The patients in this case showed fast, beneficial, symptom-related, and radiological reaction.

The name CLIPPERS suggests a principally pontine lesion and enhancement pattern; nonetheless, extrapontine implications can also be presented. The origin of CLIPPERS has yet to be discovered. Scientific researchers have suggested a precise inflammatory process or an immune-related perivascular/vascular inflammatory response (18-20).

Several clinical features and one neuroimaging element should be taken into consideration to generate a correct diagnosis: signs and symptoms related to the brainstem, commonly ataxia, dysarthria, and diplopia; an amelioration using steroid treatment and degradation with subsequent of withdrawal of treatment; MRI showing punctate similar to 'salt and pepper', predominantly located in the pons; and contrast enhancement (13,20-23).

The neuropathologic characteristics of CLIPPERS include perivascular and parenchymal lymphocytic infiltrates correlated with macrophages; microglia; and infrequently, plasma cells or neutrophils (20-23). Demyelination is absent. CD4-positive cells are more frequently encountered in comparison with CD8-positive lymphocytes or CD-20-positive B cells (22-26).

\section{Additional information}

This immunotherapy-responsive disorder is characterized by the punctate, curvilinear, perivascular gadolinium enhancement peppering the pons, with the spread of the disease often located in the cerebellar peduncles and hemispheres, caudally into the spinal cord and rostrally into the basal ganglia if present (26-30).

Symptomatology is mirrored by the characteristics of neuroimaging: facial paresthesia, diplopia, gait ataxia, and myelopathy. The clinical symptoms and signs, and the neuroimaging impairments, appear to be highly susceptible to high-dose corticosteroid treatments, but often will reappear or 'relapse' upon stopping corticoid treatment (27-30).

Immunopathological investigations of patients with CLIPPERS to date have revealed a peculiar but nonspecific, strict perivascular T cell-predominant infiltrate, which is not clear pathological proof for distinguishing diagnosis from other studied demyelinating or inflammatory diseases. Except for continuous follow-up examinations, these patients often do not have access to alternative diagnoses (26-30).

Patients who do not present clearly visible and sustained improvement clinically, symptomatically, and in neuroimaging after treatment with corticosteroids and immunosuppressants should be considered for an alternative diagnosis.

In the literature, brain biopsies have been recommended; nonetheless, this option is problematic when taking into consideration that the CLIPPERS pathology is frequently found in significant brain areas (brainstem, cerebellar peduncles, and spinal cord), with the possibility of biopsy complications $(10,26-30)$.

Moreover, although the pathological findings reported to date in patients with CLIPPERS are different from MS, sarcoidosis, glioma, and lymphoma, they are not specific.

In the future, investigations should focus on a better definition of immunopathological, radiological, and serological findings that distinguish this syndrome from other inflammatory CNS disorders. Cytokine and chemokine tests of serum and cerebrospinal fluid can provide information focusing on the mechanisms of inflammation. CLIPPERS may be an autoimmune disorder.

Immunological studies of serum and cerebrospinal fluid are ideal for considering a broader analysis of the antibodies targeting the perivascular region that may be pathogenic or may cause cell-mediated lesions (26-30).

One theory suggests that CLIPPERS could be the result of certain systemic diseases (such as Sjogren's syndrome) with heterogeneous causes, but no data currently support such a possibility (25-27).

Kastrup et al (20) documented elevated serum IgE levels in two of the three patients with CLIPPERS. They interpreted this increase in $\mathrm{IgE}$ as an 'allergic trigger as a pathophysiological link to an inflammatory vascular process'. A significant number of CLIPPERS researchers have utilized standard clinical magnetic resonance imaging to assess CLIPPERS and focused mainly on T1-weighted post-gadolinium imaging (27-30).

Although there are extensive blood-brain barrier disorders in patients with CLIPPERS, there is insufficient evidence for the presence of edema. Some researchers have reported the results of magnetic resonance spectroscopy and infusionweighted imaging, but again, no specific abnormalities were found (10,30,31). Future studies can more specifically elucidate key anomalies of this disorder, using advanced techniques, such as improving the dynamic contrast of susceptibility $(10,30,31)$.

In the literature, a possible relationship between stroke and CLIPPERS has been suggested (31-33). Although the characteristic histological features of vasculitis are not present in this syndrome, transmural lymphocytic infiltrates and inflammatory vessel occlusion have been shown to be present in biopsy samples in patients with CLIPPERS, suggesting an overlap 
or association with small vessel vasculitis (31-33). Stroke due to atherosclerotic disease of small vessels in the vulnerable post-inflammatory brainstem is a plausible physiological explanation for this theory.

\section{Conclusions}

Although CLIPPERS is a pathological syndrome that benefits from steroid treatment, it is not a benign condition. In studies reported in the literature, no patient has fully recovered, and without chronic immunosuppression, the disease can recur (33-35). Marked cerebellar atrophy accompanies or follows onset of the disease and can be measured by clinical parameters and MRI. This factor may also contribute to long-term neurological morbidity. Although the exact pathophysiological mechanisms of the disease are unclear, there appear to be clinical overlaps with other predominant autoimmune encephalitis of the brainstem $(35,36)$. Neurologists should be aware of the typical conditions and manifestations, as prompt and appropriate treatment may limit the permanent neurological effects. In differential diagnoses of neurodegenerative diseases and of vascular diseases (37-42) with similar CT and MRI images, CLIPPERS should be considered.

The pathogenesis of CLIPPERS has not yet been elucidated. The slightly elevated levels of CSF proteins without significant pleocytosis, the increased contrast in MRI lesions, a good response to steroids, and the $\mathrm{T}$ cell infiltrates observed in pathological studies suggest that it is an immune-mediated disease $(35,36)$. Although there is no specific biomarker, careful exclusion of other conditions is needed to diagnose CLIPPERS.

Clinicians are advised to take this disorder into consideration in the diagnostic area, taking into consideration that diagnosis and treatment can be followed by better long-term functional results. We need more data to understand the diagnosis and prognostic value of a negative, persistent or reverse profile of oligoclonal bands in CLIPPERS.

\section{Acknowledgements}

Not applicable.

\section{Funding}

No funding was received.

\section{Availability of data and materials}

Not applicable.

\section{Authors' contributions}

ADA, AEG and AZS conceived and designed the review. AZS, AEG, CM and ADA performed the literature research. ADA, AEG, AZS, CF, CM and DDA were involved in the interpretation of the findings in the literature. ADA, AZS, AEG, DCJ, $\mathrm{CF}$ and DDA were involved in the writing of the manuscript. All authors have read and approved the final manuscript for publication.

\section{Ethics approval and consent to participate}

Not applicable.

\section{Patient consent for publication}

Not applicable.

\section{Competing interests}

The authors declare that they have no competing interests.

\section{References}

1. Pittock SJ, Debruyne J, Krecke KN, Giannini C, van den Ameele J, De Herdt V, McKeon A, Fealey RD, Weinshenker BG, Aksamit AJ, et al: Chronic lymphocytic inflammation with pontine perivascular enhancement responsive to steroids (CLIPPERS). Brain 133: 2626-2634, 2010.

2. Kira J: The expanding phenotype of CLIPPERS: Is it a disease or a syndrome? J Neurol Neurosurg Psychiatry 83: 2-3, 2012.

3. Jones JL, Dean AF, Antoun N, Scoffings DJ, Burnet NG and Coles AJ: 'Radiologically compatible CLIPPERS' may conceal a number of pathologies. Brain 134: e187, 2011.

4. De Graaff HJ, Wattjes MP, Rozemuller-Kwakkel AJ, Petzold A and Killstein J: Fatal B-cell lymphoma following chronic lymphocytic inflammation with pontine perivascular enhancement responsive to steroids. JAMA Neurol 70: 915-918, 2013.

5. Espinosa G, Font J, Tàssies D, Vidaller A, Deulofeu R, LópezSoto A, Cervera R, Ordinas A, Ingelmo $\mathrm{M}$ and Reverter JC: Vascular involvement in Behçet's disease: Relation with thrombophilic factors, coagulation activation, and thrombomodulin. Am J Med 112: 37-43, 2002.

6. Saigal G and Quencer R: Acute lacunar infarcts in CLIPPERS: Is the chronic infiltrative lymphocytic perivascular disease process to blame? Neuroradiol J 26: 629-635, 2013.

7. Taieb G, Duflos C, Renard D, Audoin B, Kaphan E, Pelletier J, Limousin N, Tranchant C, Kremer S, de Sèze J, et al: Long-term outcomes of CLIPPERS (chronic lymphocytic inflammation with pontine perivascular enhancement responsive to steroids) in a consecutive series of 12 patients. Arch Neurol 69: 847-855, 2012.

8. Trejo-Gabriel-Galán JM, Arenaza-Basterrechea N and SedanoTous MJ: Chronic lymphocytic inflammation with pontine perivascular enhancement responsive to steroids (CLIPPERS syndrome). Med Clin (Barc) 144: 187-188, 2015 (In Spanish).

9. Altintas A, Alpaslan BG and Uygunoglu U: CLIPPERS syndrome: Case report. J Neuroimmunol 275: 413, 2014.

10. Simon NG, Parratt JD, Barnett MH, Buckland ME, Gupta R, Hayes MW, Masters LT and Reddel SW: Expanding the clinical, radiological and neuropathological phenotype of chronic lymphocytic inflammation with pontine perivascular enhancement responsive to steroids (CLIPPERS). J Neurol Neurosurg Psychiatry 83: 15-22, 2012.

11. Biotti D, Deschamps R, Shotar E, Maillart E, Obadia M, Mari I, Savatovsky J and Gout O: CLIPPERS: Chronic lymphocytic inflammation with pontine perivascular enhancement responsive to steroids. Practical Neurol 11: 349-351, 2011.

12. Blaabjerg M, Ruprecht K, Sinnecker T, Kondziella D, Niendorf T, Kerrn-Jespersen BM, Lindelof M, Lassmann H, Kristensen BW, Paul $\mathrm{F}$ and Illes Z: Widespread inflammation in CLIPPERS syndrome indicated by autopsy and ultra-high-field 7T MRI. Neurol Neuroimmunol Neuroinflamm 3: 226, 2016.

13. Kerrn-Jespersen BM, Lindelof M, Illes Z, Blaabjerg M, Lund EL, Klausen C, Christiansen I, Sellebjerg F and Kondziella D: CLIPPERS among patients diagnosed with non-specific CNS neuroinflammatory diseases. J Neurol Sci 343: 224-227, 2014.

14. Buttmann M, Metz I, Brecht I, Brück W and Warmuth-Metz M: Atypical chronic lymphocytic inflammation with pontocerebellar perivascular enhancement responsive to steroids (CLIPPERS), primary angiitis of the CNS mimicking CLIPPERS or overlap syndrome? A case report. J Neurol Sci 324: 183-186, 2013.

15. Cipriani VP, Arndt N, Pytel P, Reder AT and Javed A: Effective treatment of CLIPPERS with long-term use of rituximab. Neurol Neuroimmunol Neuroinflamm 5: e448, 2018 
16. Duprez TP and Sindic CJM: Contrast-enhanced magnetic resonance imaging and perfusion-weighted imaging for monitoring features in severe CLIPPERS. Brain 134: e184, 2011.

17. Gabilondo I, Saiz A, Graus F and Villoslada P: Response to immunotherapy in CLIPPERS syndrome. J Neurol 258: 2090-2092, 2011.

18. Izquierdo AG, Muñoz F, Valenzuela R, Gejman R and Raue M: A chilean case of chronic lymphocytic inflammation with pontine perivascular enhancement responsive to steroids (clippers): Case report. J Neurol Sci 357: 76-78, 2015.

19. Ha AD, Parratt JD, Babu S, Kim SD, Mahant N, O'Neill J, Halpern JP and Fung VS: Movement disorders associated with CLIPPERS. Mov Disord 29: 148-150, 2014.

20. Kastrup O, van de Nes J, Gasser T and Keyvani K: Three cases of CLIPPERS: A serial clinical, laboratory and MRI follow-up study. J Neurol 258: 2140-2146, 2011.

21. Kleinschmidt-DeMasters BK and West M: CLIPPERS with chronic small vessel damage: More overlap with small vessel vasculitis? J Neuropathol Exp Neurol 73: 262-267, 2014

22. Lefaucheur R, Bourre B, Ozkul-Wermester O, Maltetel D and Wallon D: Stroke mimicking relapse in a patient with CLIPPERS syndrome. Acta Neurol Belg 115: 735-736, 2015.

23. Limousin N, Praline J, Motica O, Cottier JP, Rousselot-Denis C, Mokhtari K, Gonzalez-Aguilar A and De Toffol B: Brain biopsy is required in steroid-resistant patients with chronic lymphocytic inflammation with pontine perivascular enhancement responsive to steroids (CLIPPERS). J Neurooncol 107: 223-224, 2012.

24. Marinho PB, Montanaro VV, Barroso Freitas MC and Alvarenga RM: CLIPPERS syndrome: Case report in a Brazilian patient with a long term disease evolution. Mult Scler Relat Disord 4: 311-314, 2015.

25. Mélé N, Guiraud V, Labauge P, Oppenheim C, Mas JL and Taieb G: Effective antituberculous therapy in a patient with CLIPPERS: New insights into CLIPPERS pathogenesis. Neurol Neuroimmunol Neuroinflamm 1: e6, 2014.

26. Moreira I, Cruto C, Correia C, Alves JE, Taipa R and Pires MM: Chronic lymphocytic inflammation with pontine perivascular enhancement responsive to steroids (CLIPPERS): Postmortem findings. J Neuropathol Exp Neurol 74: 186-190, 2015

27. Nakamura R, Ueno Y, Ando J, Matsuda H, Masuda A, Iiduka K, Shingai N, Takanashi M, Yokoyama K, Komatsu N and Hattori N: Clinical and radiological CLIPPERS features after complete remission of peripheral T-cell lymphoma, not otherwise specified. J Neurol Sci 364: 6-8, 2016.

28. Ortega MR, Usmani N, Parra-Herran C, Adams DJ, Steingo B and Rammohan KW: CLIPPERS complicating multiple sclerosis causing concerns of CNS lymphoma. Neurology 79: 715-716, 2012.

29. Pesaresi I, Sabato M, Desideri I, Puglioli M, Moretti P and Cosottini M: 3.0T MR investigation of CLIPPERS: Role of susceptibility weighted and perfusion weighted imaging. Magn Reson Imaging 31: 1640-1642, 2013.
30. Sempere AP, Mola S, Martin-Medina P, Bernabeu A, Khabbaz E and Lopez-Celada S: Response to immunotherapy in CLIPPERS: Clinical, MRI, and MRS follow-up. J Neuroimaging 23: 254-255, 2013.

31. Suer D, Yusifova L, Arsava EM, Ekinci G, Us O and Uluc K: A case report of CLIPPERS (Chronic Lymphocytic Inflammation with Pontocerebellar Perivascular Enhancement Responsive to Steroids) syndrome. Clin Neuroradiol 25: 61-63, 2015

32. Tan BL, Agzarian M and Schultz DW: CLIPPERS: Induction and maintenance of remission using hydroxychloroquine. Neurol Neuroimmunol Neuroinflamm 2: e56, 2015.

33. Zhang YX, Hu HT, Ding XY, Chen LH, Du Y, Shen CH, Guo Y and Ding MP: CLIPPERS with diffuse white matter and longitudinally extensive spinal cord involvement. Neurology 86 : 103-105, 2016.

34. Wijntjes J, Wouda EJ, Siegert CE, Karas GB and Vlaar AM: Need for prolonged immunosupressive therapy in CLIPPERS-a case report. BMC Neurol 13: 49, 2013.

35. Wetter A, Ku peli G, Meila D, Brassel F and Nacimiento W: CLIPPERS syndrome in a patient with a fluctuating cerebellar syndrome. Neurographics 3: 11-13, 2013.

36. Weng CF, Chan DC, Chen YF, Liu FC and Liou HH: Chronic hepatitis B infection presenting with chronic lymphocytic inflammation with pontine perivascular enhancement responsive to steroids (CLIPPERS): A case report. J Med Case Rep 9: 266 , 2015.

37. Gogu A: New clinical-imagistic features in cerebral venous and sinus thrombosis. Procedia Soc Behav Sci 191: 1650-1659, 2015.

38. Gogu AE, Pusztai A, Stroe AZ, Docu Axelerad D and Docu Axelerad A: Back pain in rare diseases: A comparison of neck and back pain between spinal cord ischemia and spinal dural arteriovenous fistula. Brain Sci 10: 618, 2020.

39. Jianu DC, Jianu SN, Motoc AGM, Poenaru M, Petrica L, Vlad A, Ursoniu S, Gogu AE and Dan F: Diagnosis and management of a young woman with acute isolated lateral sinus thrombosis. Rom J Morphol Embryol 58: 1515-1518, 2017.

40. Gogu A, Lupu M and Axelerad AD: The relationship between ischemic stroke and atrial fibrillation. Procedia Soc Behav Sci 197: 2004-2010, 2015 .

41. Gogu AE, Jianu DC, Dumitrascu V, Ples H, Stroe AZ, Docu Axelerad D and Docu Axelerad A: MTHFR gene polymorphisms and cardiovascular risk factors, clinical-imagistic features and outcome in cerebral venous sinus thrombosis. Brain Sci 11: 23, 2020.

42. Gogu AE, Motoc AG, Stroe AZ, Docu Axelerad A, Docu Axelerad D, Petrica L and Jianu DC: Plasminogen activator inhibitor-1 (PAI-1) gene polymorphisms associated with cardiovascular risk factors involved in cerebral venous sinus thrombosis. Metabolites 11: 266, 2021. 